\title{
Balancing between bleeding and thromboembolism after percutaneous coronary intervention in patients with atrial fibrillation. Could triple anticoagulant therapy be a solution?
}

\author{
Magdalena Dąbrowska ${ }^{1}$, Andrzej Ochała ${ }^{2}$, Wiesław Cybulski ${ }^{3}$, Michał Tendera ${ }^{3}$ \\ ${ }^{1}$ Cardiology Student Research Group, $3^{\text {rd }}$ Division of Cardiology, $3^{\text {rd }}$ Department of Cardiology, Medical University of Silesia, \\ Katowice, Poland \\ IInvasive Cardiology Department, Medical University of Silesia, Katowice, Poland \\ $3_{3}^{\text {rd }}$ Division of Cardiology, $3^{\text {rd }}$ Department of Cardiology, Medical University of Silesia, Katowice, Poland
}

Postep Kardiol Inter 2013; 9, 3 (33): 234-240

DOI: $10.5114 /$ pwki.2013.37501

\begin{abstract}
Introduction: Atrial fibrillation (AF) has nowadays become a common disease as it comes along with medical procedures propagation in the ageing population with coexistent diseases. Hence a need for use of combined anticoagulant and antithrombotic therapy has arisen. According to the 2010 ESC guidelines on myocardial revascularization, short-term triple antithrombotic therapy after percutaneous coronary intervention $(\mathrm{PCl}$ ) should be given if compelling indications exist.

Aim: To assess bleeding and thromboembolic events depending on the antithrombotic regimen in short- and long-term followup in patients with $\mathrm{AF}$ after $\mathrm{PCl}$ with stent implantation.

Material and methods: A 12-month prospective, non-randomized registry was conducted in the $3^{\text {rd }}$ Department of Cardiology in the Upper Silesian Medical Center in Katowice from October 2008 to April 2011. One hundred and four patients in two groups - on triple therapy (TT; aspirin + clopidogrel + vitamin K antagonists (VKA; warfarin or acenocoumarol) $n=44)$ and on dual therapy (DT; aspirin + clopidogrel; $n=60$ ) - were assessed 30 days and 12 months after angioplasty.

Results: All bleeding events occurred more often in the triple anticoagulated group in 30 days (TT 20.5\% vs. DT 6.7\%; $p=0.03$ ) and after 12 months (TT 38.9\% vs. DT 17.2\%, $p=0.09$ ). The difference in major bleeding events was not significant after 30 days (TT $9.1 \%$ vs. DT $3.3 \%$; $p=$ NS) or 12 months (TT $11.1 \%$ vs. DT $6.9 \%$; $p=$ NS). Thromboembolic events after 30 days (DT $5.0 \%$ vs. TT $2.3 \%$ ) and 12 months (TT $11.1 \%$ vs. DT 3.4\%) were comparable. The percentage of deaths after 30 days (DT $1.7 \%$ vs. TT $0.0 \%, p=$ NS) increased after 12 months (DT $13.8 \%$ vs. TT $0.0 \%, p=0.09$ ).

Conclusions: Significantly higher risk of bleeding on TT becomes blurred by a tendency to increased mortality in patients on DT.
\end{abstract}

Key words: anticoagulants, percutaneous coronary intervention, atrial fibrillation, triple therapy.

\section{Introduction}

The use of dual antiplatelet therapy with aspirin and thienopyridine has become a gold standard in prevention of coronary artery stent thrombosis after percutaneous coronary intervention $(\mathrm{PCI})[1,2]$. Simultaneously, oral anticoagulant therapy $(\mathrm{OAC})$ is recommended when dealing with atrial fibrillation [3, 4], mechanical heart valves [5] and venous thromboembolism [6]. Atrial fibrillation has nowadays become a common disease as it comes along with medical procedures propagation in the ageing population with coexistent diseases. According to the 2010 ESC guidelines on myocardial revascularization [7], short-term triple anti- thrombotic therapy should be given if compelling indications exist. In effect we approach the problem of increased risk of both bleeding and thromboembolism. There are limited data on safety and efficacy of triple therapy in patients after $\mathrm{PCl}$ and stent implantation.

\section{Aim}

The aim of our registry was to assess bleeding and embolic events depending on the antithrombotic regimen in long-term follow-up in patients with atrial fibrillation after $\mathrm{PCl}$ with stent implantation. 


\section{Material and methods}

The 12-month prospective, non randomized registry was conducted in the $3^{\text {rd }}$ Department of Cardiology in the Upper Silesian Medical Center in Katowice from October 2008 to April 2011.

Consecutive patients with paroxysmal, persistent or permanent non-valvular atrial fibrillation qualified for invasive treatment with stent implantation (either bare metal stents (BMS) or drug-eluting stents (DES)) were enrolled in the registry $(n=104)$. Patients were assessed one month and one year after angioplasty. All major adverse cardiovascular and cerebrovascular events, especially bleeding and thromboembolic events, were registered, and also whether they required dose change or discontinuation of any of the three medications. We assessed drug compliance and noted hospitalizations due to bleeding or thrombotic events during the period in which they were treated with all three drugs. Exclusion criteria were: contraindications to medications, lack of patient's consent and high likelihood of nonadherence to the therapy.

\section{Statistical analysis}

Statistical analysis was performed with the program 'Statistica 8'. Continuous variables are presented as means plus or minus $1 \mathrm{SD}$. Discrete variables are presented as percentages. The $\chi^{2}$ test was used to compare discrete variables and the Student $t$ test to compare continuous variables. A two-sided $p$-value of $<0.05$ was considered to be statistically significant.

\section{Results}

One hundred and four consecutive patients were enrolled (Figure 1). Patients were divided into two groups: on triple therapy (TT; aspirin + clopidogrel + vitamin K antagonists (VKA; warfarin or acenocumarol); $n=44$ ) and on dual therapy (DT; aspirin + clopidogrel; $n=60$ ). Baseline characteristics are given in Table 1 . The two study groups differed significantly according to previous coronary artery bypass surgery, which was more frequent in the group receiving oral anticoagulants ( $29 \%$ vs. $10 \%, p=0.01)$. Additionally, the incidence of diabetes mellitus was higher in this group ( $57 \%$ vs. $28 \%, p=0.003$ ), and mean left ventricle ejection fraction was significantly lower ( $45 \pm 11 \%$ vs. $50 \pm 12 \%, p=0.03$ ).

Antithrombotic drugs were administered in standard recommended doses. Groups were assessed after 1 month (short-term observation) and 1 year (long-term observation). The use of glycoprotein IIb/IIla inhibitors was at the discretion of the operator.

In the TT group 18 patients continued triple therapy for at least 12 months, 26 patients discontinued one of the

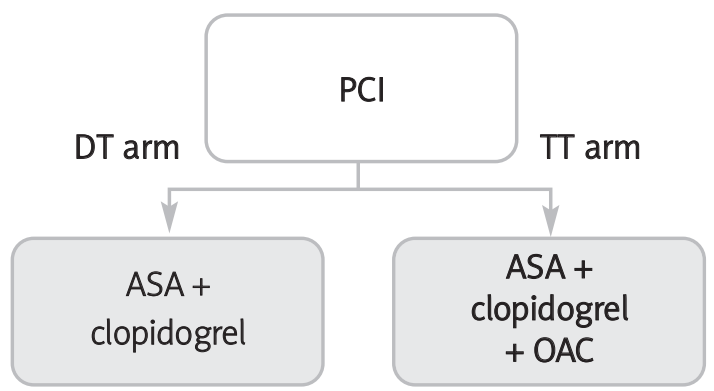

30 days observation $(n=104)$

12 months observation $(n=47)$

Fig. 1. Study design

Table 1. Baseline characteristics

\begin{tabular}{|c|c|c|c|}
\hline Characteristic & Group A $(n=44)$ & Group B $(n=60)$ & Value of $p$ \\
\hline Age [years] & $69 \pm 7$ & $71 \pm 9$ & 0.29 \\
\hline Gender, $n$ (\%) (female) & $15(34)$ & $28(47)$ & 0.19 \\
\hline $\operatorname{LVEF}[\%](n=95)$ & $45 \pm 11$ & $50 \pm 12$ & 0.03 \\
\hline GFR $\left[\mathrm{ml} / \mathrm{min} / 1.73 \mathrm{~m}^{2}\right]$ & $69 \pm 16$ & $74 \pm 23$ & 0.18 \\
\hline pMI, $n(\%)$ & $19(43)$ & $24(40)$ & 0.74 \\
\hline $\mathrm{pPCl}, n(\%)$ & $19(43)$ & $21(35)$ & 0.39 \\
\hline pCABG, $n(\%)$ & $13(29)$ & $6(10)$ & 0.01 \\
\hline Diabetes, $n(\%)$ & $25(57)$ & $17(28)$ & 0.003 \\
\hline Hypertension, $n(\%)$ & $41(93)$ & $51(85)$ & 0.19 \\
\hline Dyslipidemia, $n(\%)$ & $43(98)$ & $57(95)$ & 0.13 \\
\hline GP IIb/IIla antagonists, $n$ (\%) & $4(9.1)$ & $5(8.3)$ & 0.89 \\
\hline Drug-eluting stents, $n$ (\%) & $12(27)$ & $13(22)$ & 0.47 \\
\hline Previous stroke/TIA, $n$ (\%) & $5(11.4)$ & $5(8.3)$ & 0.60 \\
\hline $\mathrm{IPP} / \mathrm{H}_{2} \mathrm{~B}, n(\%)$ & $20(45)$ & $32(53)$ & 0.42 \\
\hline
\end{tabular}

$L V E F$ - left ventricle ejection fraction, GFR - glomerular filtration rate from MDRD, $\mathrm{pMI}$ - previous myocardial infarction, $\mathrm{pPCl}$ - previous percutaneous coronary intervention, $P C A B G$ - previous coronary artery bypass grafting, $T I A$ - transient ischemic attack, IPP/ $\mathrm{H}_{2} B-$ proton-pump inhibitor/ $\mathrm{H}_{2}$-blocker 
drugs (generally an antithrombotic drug) either after 1 month (4 patients) or after 3 months (22 patients). Therefore 18 patients from that group met the long-term triple therapy observation criteria.

In the DT group 29 out of 60 patients took drugs for 12 months and longer, 18 for up to 12 months, and 7 patients were on dual therapy for only 1 month. Six patients from this group were lost to 1-year follow-up (Figure 2).

The primary end-points were: safety and efficacy of administered therapy. Secondary end-points were: bleeding and thromboembolic events, death from any cause, recurrent acute coronary syndrome and stent thrombosis. Major bleeding was defined as bleeding requiring hospitalization and/or discontinuation of any antithrombotic medication. All other bleeding events were considered as minor bleeding. The risk of bleeding was estimated by means of GRACE registry predictors [8] and modified HAS-BLED risk score [9]. Thromboembolic risk was rated using $\mathrm{CHADS}_{2}$ [10] and $\mathrm{CHA}_{2} \mathrm{DS}_{2}$-VaSC [11] scores.

Bleeding risk was estimated using the GRACE registry. This included parameters such as advanced age ( $\geq 75$ years old), female sex, renal insufficiency, bleeding history, use of glycoprotein Ilb/Illa antagonists antagonists and percutaneous coronary intervention (Figure 3). Similarly, we used the HAS-

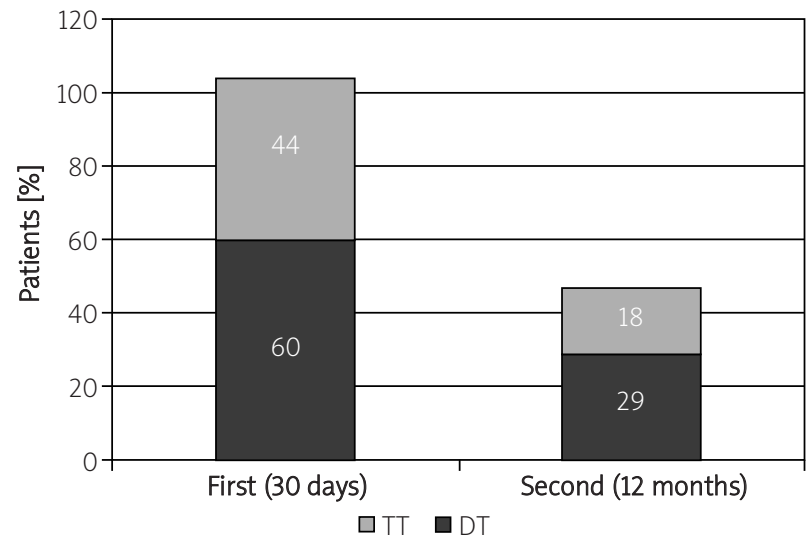

Fig. 2. Number of patients regarding cut-off points

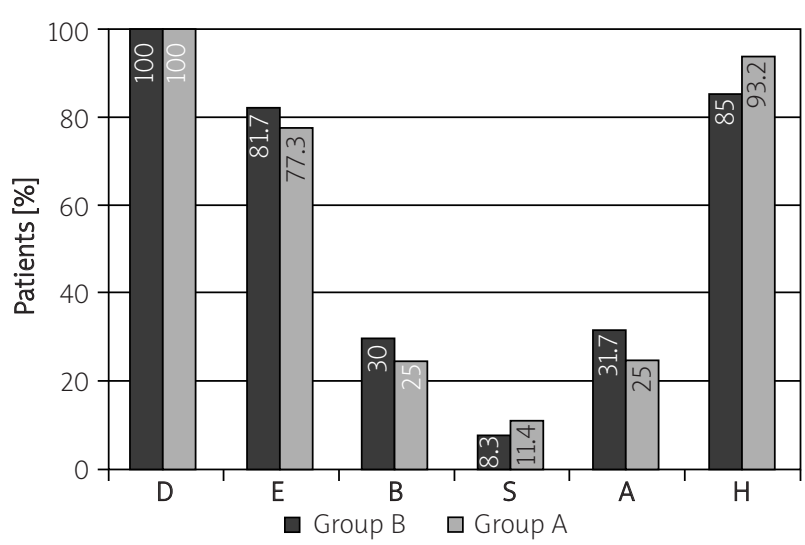

Fig. 4. Modified HAS-BLED bleeding risk score $(p=N S)$
BLED score to analyze bleeding risk, omitting international normalized ratio (INR) parameter however (Figure 4). This was due to lack of this parameter in the DT group, as well as insufficient data in triple therapy patients. The modified HAS-BLED score was composed of the following parameters: hypertension, abnormal renal/liver function, stroke, bleeding, elderly age, drugs or alcohol. There were no significant differences between the two groups concerning their bleeding risk in these scales.

For thromboembolic risk, we used $\mathrm{CHADS}_{2}$ (Figure 5) and $\mathrm{CHA}_{2} \mathrm{DS}_{2}$-VASc (Figure 6) scores. Both scales revealed differences when assessing diabetes incidence $(p=0.03)$. The global percentage of strokes measured with $\mathrm{CHADS}_{2}$ (9.6\%) and $\mathrm{CHA}_{2} \mathrm{DS}_{2}$-VASc (14.4\%) scores differed owing to widened stroke criteria in the latter score. The mean $\mathrm{CHADS}_{2}$ level was $2.12 \pm 1.18$. $95 \%$ of patients $(n=99)$ were classified in the medium- or high-risk category; those in the highrisk category were more often on triple antithrombotic therapy (TT 43.2\% vs. DT 23.3\%; $p=0.03$ ), whereas medium risk was associated with double therapy (DT 70.0\% vs. TT 54.5\%; $p=\mathrm{NS}$ ) (Figure 7). Although overall $\mathrm{CHADS}_{2}$ subgroups' risk level differed (DT $1.85 \pm 1.15$ vs. TT $2.39 \pm 1.22$; $p=0.02$ ), significance blurred in CHA2DS2-VASc estimation (DT $3.98 \pm 1.87$ vs. TT $4.36 \pm 1.51 ; p=N S$ ).

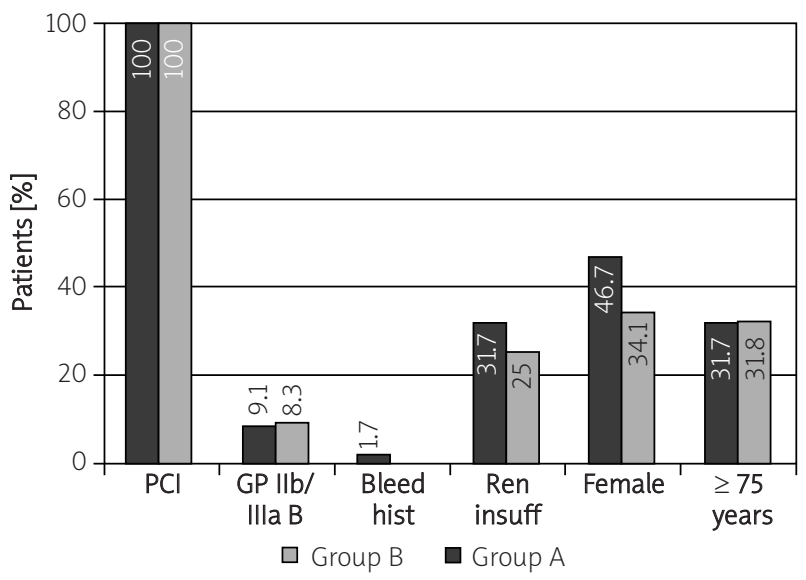

Fig. 3. GRACE bleeding risk score $(p=N S)$

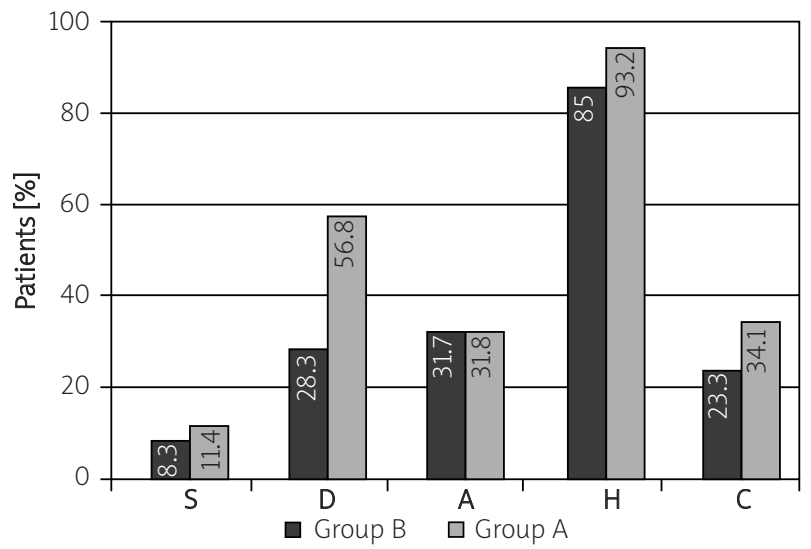

Fig. 5. $\mathrm{CHADS}_{2}$ risk score $(p=0.03)$ 


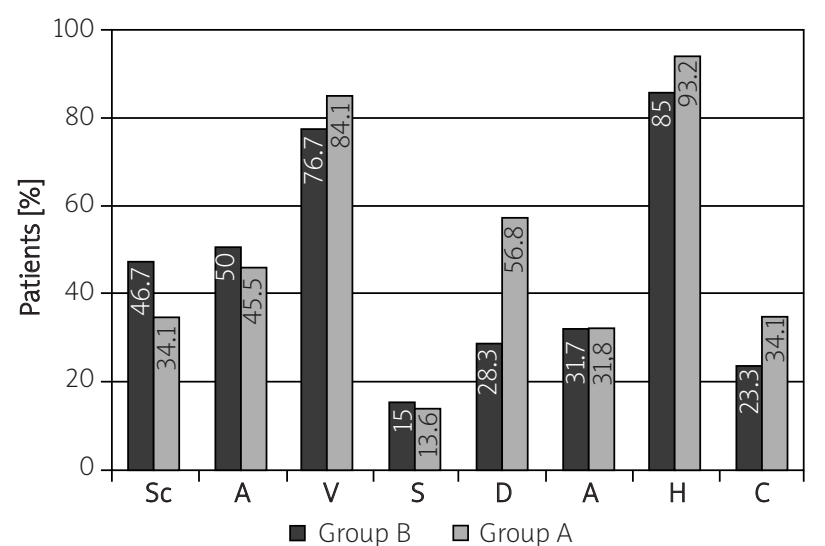

Fig. 6. $\mathrm{CHA}_{2} \mathrm{DS}_{2}$-VASc risk score $(p=0.03)$

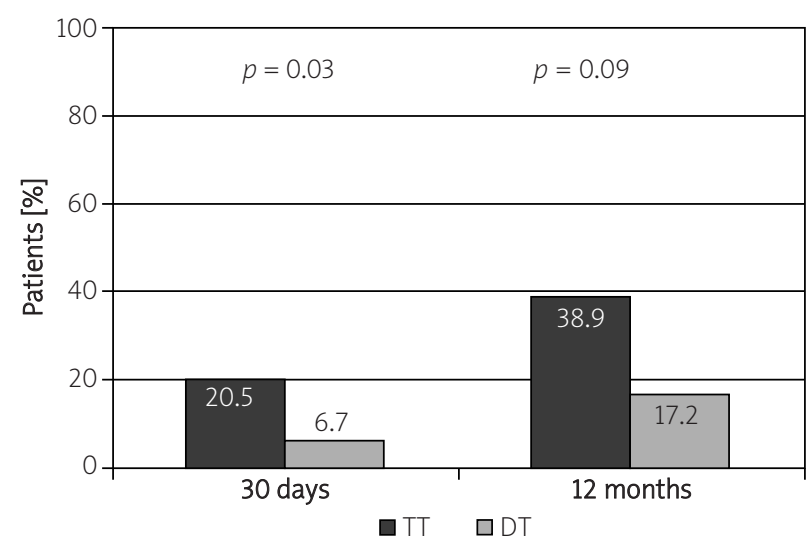

Fig. 8. All bleeding events

A significant bleeding risk factor was addition of VKA. It raised the chance of bleeding 3.6-fold ( $p=0.04$ ). Also, increasing of 1 point in modified HAS-BLED score caused 1.86 global increase of risk $(p=0.03)$. When analyzing only the TT group, the risk was even higher -2.84 -fold $(p=0.02)$.

All bleeding events occurred in 30 days statistically more often in the triple-anticoagulated group (TT 20.5\% vs. DT $6.7 \% ; p=0.03)$. After 12 months only a tendency was observed (TT 38.9\% vs. DT 17.2\%, $p=0.09$ ), though (Figure 8). The difference in major bleeding was not significant after either 30 days (TT 9.1\% vs. DT 3.3\%; $p=$ NS) or 12 months (TT $11.1 \%$ vs. DT $6.9 \% ; p=$ NS), or in minor bleeding after 30 days (TT $11.4 \%$ vs. DT 3.3\%; $p=\mathrm{NS}$ ) or 12 months (TT $27.8 \%$ vs. DT $10.3 \%, p=$ NS).

Thromboembolic events after 30 days happened in both groups (DT 5.0\% vs. TT 2.3\%; $p=$ NS) (Figure 9). Surprisingly, after 12 months embolism was reported more often in the triple therapy group (TT $11.1 \%$ vs. DT 3.4\%; $p=\mathrm{NS}$ ).

Regarding secondary end-points, four deaths occurred during 12-month observation, among them one early (Figure 10). The percentage after 30 days (DT 1.7\% vs. TT 0.0\%, $p=\mathrm{NS}$ ) increased after 12 months (DT $13.8 \%$ vs. TT $0.0 \%$, $p=0.09$ ). Of note, deaths occurred only in the group on

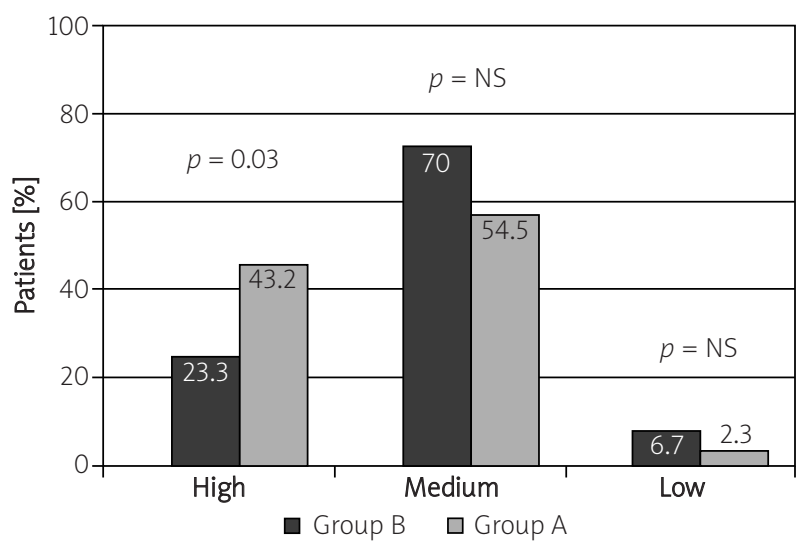

Fig. 7. $\mathrm{CHADS}_{2}$ risk level $(p=0.03)$

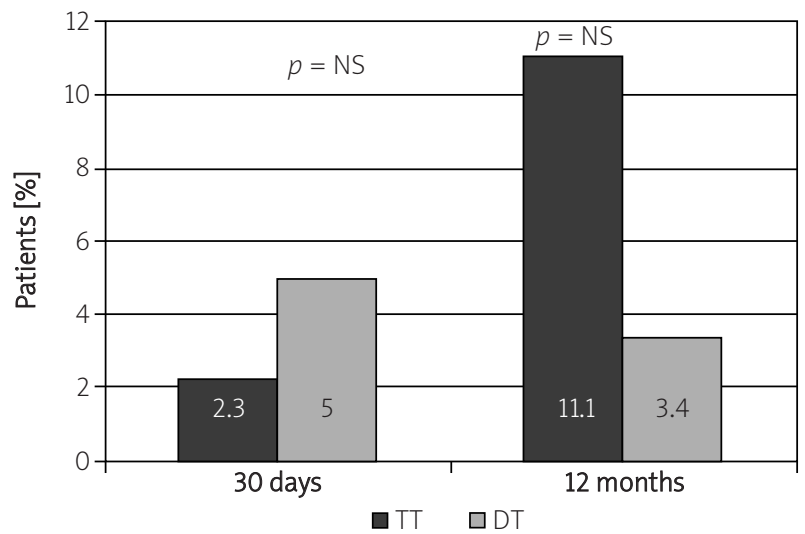

Fig. 9. Thromboembolism

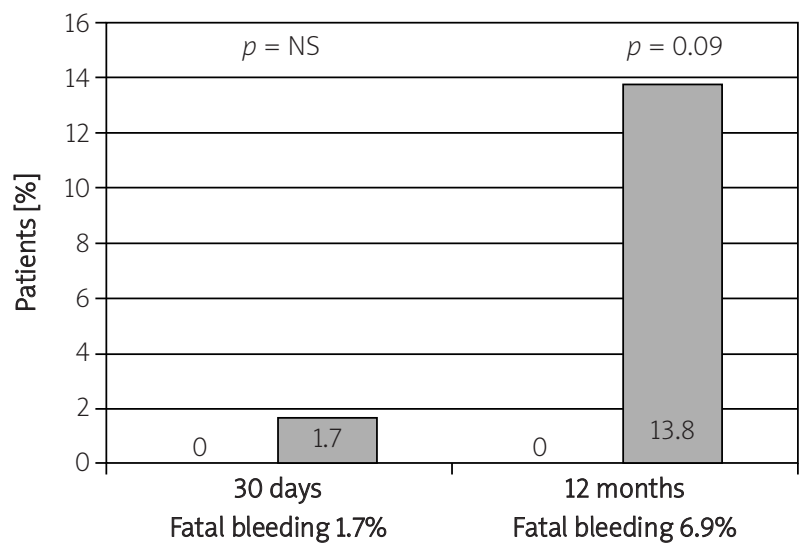

Fig. 10. Deaths

double therapy. Two deaths were due to fatal bleeding; one of them was periprocedural. Both were the result of gastrointestinal bleeding.

Recurrent acute coronary syndrome was more frequent in the group without VKA after 12 months (DT $11.1 \%$ vs. TT $3.4 \%, p=\mathrm{NS}$ ). There were no such incidences after 30 days in the DT or TT group. We did not observe stent thrombosis any time in any group. 


\section{Discussion}

As we know from previous studies, triple therapy is reasonable in patients with atrial fibrillation who underwent $\mathrm{PCl}$, because OACs prevent stroke more efficiently than ASA with thienopyridines [12], but OACs with aspirin do not prevent stent thrombosis as well as thienopyridines [13]. The dilemma is how to order triple therapy individually to equilibrate bleeding and thromboembolic risk and achieve compelling guidelines to comply with. Some factors increase both bleeding and thromboembolic risk [14], and therefore individual assessment of patients is vital.

The global $\mathrm{CHADS}_{2}$ risk level showed that $95 \%$ of cases were estimated between intermediate and high risk of thromboembolism and had compelling indications to be triple-anticoagulated [15]. According to the differences in baseline characteristics and $\mathrm{CHADS}_{2}$ risk between DT and TT groups, in this non-randomized study OACs were given to patients with more comorbidities. It may place them at higher risk for anticoagulant complications, but they also have characteristics that make them more likely to benefit from that regimen.

The main concern of triple anticoagulant therapy is bleeding. It is a difficult task to estimate the factual bleeding risk, as a lot of parameters differ among studies - indications, medicines, doses, duration of therapy, baseline characteristics, diet, risk scores, bleeding criteria. Additionally, compliance of patients differs in a wide range. The significantly higher bleeding incidence after 30 days in the TT group was not sustained after 12 months, probably due to the small group of patients. However, it confirmed the results from previous studies on the impact of VKA on bleeding $[16,17]$. A large registry of almost 120,000 patients with atrial fibrillation indicates the yearly bleeding risk of triple antithrombotic therapy at the level of $16 \%$ [18]. Our registry indicated bleeding incidents in $20.5 \%$ after 30 days and $38.9 \%$ after 12 months. Up-to-date WOEST Study results show that discontinuation of aspirin reduces bleeding events without raising thromboembolic events. This can be one solution in the group of atrial fibrillation patients after PCI procedures.

The percentage of serious bleeding events in the group on triple therapy after 30 days (9.1\%) and within 12 months $(11.1 \%)$ is in concert with the results of Valencia et al. [19] and Orford et al. [20], who reported $8.6 \%$ and $9.1 \%$ incidence of major bleeding, respectively. Available data present a wide range of major bleedings frequency from $4.7 \%$ [21] to $21.6 \%$ [22].

The trend in thromboembolic events after 30 days showed insufficient anticoagulation more often in DT, but after 12 months it was definitely in favor of DT. We suppose that it is influenced by the lability of the INR index in the group on VKA, as keeping this parameter stable in the therapeutic range of 2.0-3.0 can be a real challenge.

With respect to secondary end-points, a tendency towards significance in death occurrence was observed. Four deaths took place in the DT group. What is more striking, $50 \%$ of them were based on fatal bleeding. Better compliance is probably not responsible for this aspect; both bleeding and thromboembolic complications were more frequent in the TT group. Looking more closely at fatal bleeding, the estimated risk in one case was $6 / 6$ for modified HAS-BLED score, $6 / 6$ for $\mathrm{CHADS}_{2}$ and $9 / 9$ for $\mathrm{CHA}_{2} \mathrm{DS}_{2}$-VASc score. The second case presented $4 / 6,3 / 6$ and $6 / 9$ respectively. In actual fact in these cases it was a choice between wind and water. But still, the thromboembolic burden was greater in the TT group.

Some interesting trends in atrial fibrillation patients after $\mathrm{PCl}$ are available of late. Hence we are moving from an era of two oral anticoagulants to a wide range of solutions. Most importantly, alternatives to warfarin and acenocoumarol have emerged: factor Xa inhibitors (rivaroxaban, apixaban) and a direct thrombin inhibitor (dabigatran). Apixaban, according to the AVERROES [23] study, showed potential in preventing stroke. The results from the ARISTOTLE [24] trial confirmed the above. Dabigatran has favorable pharmacokinetics, and in the RE-LY trial proved to be safer and more effective in preventing thromboembolism in patients with atrial fibrillation [25]. However, there is higher risk of bleeding after addition of novel anticoagulants to the antiplatelet therapy reported in the literature (APPRAISE [26], ATLAS ACS-TIMI 46 [27], Dabigatran vs. placebo trial [28]). Thus there is a need for further studies to answer whether the group of $\mathrm{PCl}$ patients with atrial fibrillation benefit from the combination of new anticoagulants and antiplatelets.

Furthermore, the techniques to occlude the left atrial auriculum, which is the main localization of creating thrombi (PLAATO and WATCHMAN systems), appeared in the ESC guidelines (class of recommendation IIb/B). It can be an attractive therapeutic option in patients who have contraindications to anticoagulation. Next, the STENTICO Registry confirmed a safer radial than femoral approach during the PCl procedure [29]. Lastly, alternatives to clopidogrel such as prasugrel and ticagrelor are available. They have reached first class of recommendation in STEMI and NSTEMI treatment (ticagrelor) [7]. According to the TRITON TIMI 38 study [30], prasugrel is connected with higher risk of bleeding, though. Ticagrelor in the PLATO study showed comparable incidence of major bleeding. Regarding their potentially stronger impact on the coagulation system there could arise the possibility of administering aspirin + prasugrel or ticagrelor alone instead of triple therapy. There are no data on these options yet.

To sum up, the answer how to manage patients suffering from atrial fibrillation after $\mathrm{PCl}$ procedures is somewhat a sealed book. The accurate assessment of the bleeding and thromboembolic risk is essential to tailor the optimal individual anticoagulant regimen to such patients' needs.

We have to mention some limitations of the study. Firstly, it was not always possible to check the INR level when contacting the patient. Only information about INR labili- 
ty was received. It does limit interpretation of bleeding events, and therefore we assumed different criteria of bleeding than usual (e.g. GUSTO, TIMI, HORIZONS-AMI). Secondly, it was of our concern to determine the frequency of complications on authentic triple antithrombotic therapy throughout the whole period. It was not a standard procedure to implant drug-eluting stents in patients with indications for VKAs. Hence cases for long-term observation were difficult to assemble.

\section{Conclusions}

Significantly higher risk of bleeding on TT becomes blurred by a tendency to increased mortality in patients on DT. These results call for very individual therapy assessment and careful monitoring of AF patients after $\mathrm{PCl}$. Administering triple therapy in patients with high risk of thromboembolism in accordance with ESC guidelines is relatively safe, provided that there is strict cooperation between patient and doctor as well as regular and frequent monitoring of the INR parameter. Further studies are necessary to evaluate whether the new therapeutic options could be more effective and safer than current triple antithrombotic therapy.

\section{References}

1. Mehta SR, Yusuf S, Peters RJ, et al. Effects of pretreatment with clopidogrel and aspirin followed by long-term therapy in patients undergoing percutaneous coronary intervention: the PCI-CURE study. Lancet 2001; 358: 527-533.

2. Mishkel GJ, Aguirre FV, Ligon RW, et al. Clopidogrel as adjunctive antiplatelet therapy during coronary stenting. J Am Coll Cardiol 1999; 34: 1884-1890.

3. van Walraven C, Hart RG, Singer DE, et al. Oral anticoagulants vs aspirin in nonvalvular atrial fibrillation: an individual patient metaanalysis. JAMA 2002; 288: 2441-2448.

4. Hart RG, Pearce LA, Aguilar MI. Meta-analysis: antithrombotic therapy to prevent stroke in patients who have nonvalvular atrial fibrillation. Ann Intern Med 2007; 146: 857-867.

5. Butchart EG, Gohlke-Bärwolf C, Antunes MJ, et al. Recommendations for the management of patients after heart valve surgery. Eur Heart J 2005; 26: 2463-2471.

6. Hirsh J, Lee AY. How we diagnose and treat deep vein thrombosis. Blood 2002; 99: 3102-3110.

7. ESC/EACTS Guidelines on myocardial revascularization. Eur Heart J 2010; 31: 2501-2555.

8. Moscucci M, Fox KA, Cannon CP, et al. Predictors of major bleeding in acute coronary syndromes: the Global Registry of Acute Coronary Events (GRACE). Eur Heart J 2003; 24: 1815-1823.

9. Pisters R, Lane DA, Nieuwlaat R, et al. A novel user-friendly score (HAS-BLED) to assess 1-year risk of major bleeding in patients with atrial fibrillation: the Euro Heart Survey. Chest 2010; 138: 1093-1100.

10. Gage BF, Waterman AD, Shannon W, et al. Validation of clinical classification schemes for predicting stroke: results from the National Registry of Atrial Fibrillation. JAMA 2001; 285: 2864-2870.

11. Lip GY, Nieuwlaat R, Pisters R, et al. Refining clinical risk stratification for predicting stroke and thromboembolism in atrial fibrillation using a novel risk factor-based approach: the euro heart survey on atrial fibrillation. Chest 2010; 137: 263-272.
12. The ACTIVE Writing Group on behalf of the ACTIVE Investigators.Clopidogrel plus aspirin versus oral anticoagulation for atrial fibrillation in the Atrial fibrillation Clopidogrel Trial with Irbesartan for prevention of Vascular Events (ACTIVE W): a randomised controlled trial. Lancet 2006; 367: 1903-1912.

13. Leon MB, Baim DS, Popma JJ. A clinical trial comparing three antithrombotic-drug regimens after coronary-artery stenting. Stent Anticoagulation Restenosis Study Investigators. N Engl J Med 1998; 339: 1665-1671.

14. lijima R, Ndrepepa G, Mehilli J. Profile of bleeding and ischaemic complications with bivalirudin and unfractionated heparin after percutaneous coronary intervention. Eur Heart J 2009; 30: 290-296.

15. Schömig A, Sarafoff N, Seyfarth M. Triple antithrombotic management after stent implantation: when and how? Heart 2009; 95 : 1280-1285.

16. Dewilde WJ, Oirbans T, Verheugt FW, et al. WOEST study investigators. Use of clopidogrel with or without aspirin in patients taking oral anticoagulant therapy and undergoing percutaneous coronary intervention: an open-label, randomised, controlled trial. Lancet 2013; 381: 1107-1115.

17. Khurram Z, Chou E, Minutello R, et al. Combination therapy with aspirin, clopidogrel and warfarin following coronary stenting is associated with a significant risk of bleeding. J Invasive Cardiol 2006; 18: 162-164.

18. Hansen ML, Sørensen R, Clausen MT, et al. Risk of bleeding with single, dual, or triple therapy with warfarin, aspirin, and clopidogrel in patients with atrial fibrillation. Arch Intern Med 2010; 170: 1433-1441.

19. Valencia J, Mainar V, Bordes P, et al. Observance of antiplatelet therapy after stent implantation in patients under chronic oral anticoagulant treatment. J IntervCardiol 2008; 21: 218-224.

20. Orford JL, Fasseas P, Melby S, et al. Safety and efficacy of aspirin, clopidogrel, and warfarin after coronary stent placement in patients with an indication for anticoagulation. Am Heart J 2004; 147: 463-467.

21. Rogacka R, Chieffo A, Michev I, et al. Dual antiplatelet therapy after percutaneous coronary intervention with stent implantation in patients taking chronic oral anticoagulation. JACC Cardiovasc Interv 2008; 1: 56-61.

22. Manzano-Fernández S, Pastor FJ, Marín F, et al. Increased major bleeding complications related to triple antithrombotic therapy usage in patients with atrial fibrillation undergoing percutaneous coronary artery stenting. Chest 2008; 134: 559-567.

23. Connolly SJ, Eikelboom J, Joyner C, et al. Apixaban in patients with atrial fibrillation. N Engl J Med 2011; 364: 806-817.

24. Granger CB, Alexander JH, McMurray JJ, et al. Apixaban versus warfarin in patients with atrial fibrillation. N Engl J Med 2011; 365 : 981-992.

25. Connolly SJ, Ezekowitz MD, Yusuf S, et al. Dabigatran versus warfarin in patients with atrial fibrillation. N Engl J Med 2009; 361: 1139-1151.

26. Alexander JH, Becker RC, Bhatt DL, et al. Apixaban, an oral, direct, selective factor Xa inhibitor, in combination with antiplatelet therapy after acute coronary syndrome: results of the Apixaban for Prevention of Acute Ischemic and Safety Events (APPRAISE) trial. Circulation 2009; 119: 2877-2885.

27. Mega JL, Braunwald E, Mohanavelu S, et al. Rivaroxaban versus placebo in patients with acute coronary syndromes (ATLAS ACS-TIMI 46): a randomised, double-blind, phase II trial. Lancet 2009; 374: 29-38.

28. Oldgren J, Budaj A, Granger CB, et al. Dabigatran vs. placebo in patients with acute coronary syndromes on dual antiplatelet ther- 
apy: a randomized, double-blind, phase II trial. Eur Heart J 2011; 32: $2781-2789$.

29. Gilard M, Blanchard D, Helft G, et al. Antiplatelet therapy in patients with anticoagulants undergoing percutaneous coronary stenting (from STENTIng and oral antiCOagulants [STENTICO]). Am J Cardiol 2009; 104: 338-342.

30. Wiviott SD, Braunwald E, McCabe CH, et al. Prasugrel versus clopidogrel in patients with acute coronary syndromes. N Engl J Med 2007; 357: 2001-2015. 